\title{
Intensidad de demanda de los videojuegos y su efecto sobre el estado de ánimo y la activación percibida*
}

Intensity of Demand for Video Games and its Effect on Mood and Perceived Activation

Recibido: abril 30 de 2012 | Revisado: septiembre 3 de 2012 | Aceptado: octubre 28 de 2012

\author{
ROLANDO PÉREZ-SÁNCHEZ** \\ DAVID TORRES
}

Universidad de Costa Rica, San José, Costa Rica

doi:10.11144/Javeriana.UPSY13-4.idve

Para citar este artículo: Pérez-Sánchez, R., \& Torres, D. (2014). Intensidad de demanda de los videojuegos y su efecto sobre el estado de ánimo y la activación percibida. Universitas Psychologica, 13(4), 1489-1502. http://dx.doi.org/10.11144/Javeriana. UPSY13-4.idve

* Artículo de investigación.

** Instituto de Investigaciones Psicológicas. Dirección postal: 11501-2060. San José, Costa Rica. E-mails: rolarez@gmail.com,datofez1@yahoo.com

\section{RESUMEN}

Este estudio busca indagar la relación entre el uso de videojuegos con diferente intensidad de activación demandada sobre la activación fisiológica percibida y los estados de ánimo. Se utilizó un diseño de medidas repetidas $(N=27)$. Los resultados muestran que el estado de ánimo positivo aumenta ante el juego de alta activación demandada, mientras que el uso de videojuegos no afecta los estados de ánimo negativos. En cuanto a la activación fisiológica percibida, se encontró que, tanto ante el juego de baja como con el de alta activación demandada, se presenta un aumento en la activación percibida. Igualmente, se encontraron variaciones por género en el juego de baja intensidad. Se discuten las implicaciones de los resultados.

\section{Palabras clave}

videojuegos; estados de ánimo; activación fisiológica percibida; psicología de los medios de comunicación; efectos psicológicos

\section{A B S T R A C T}

This study analyzes the relationship between videogames use with different activation intensity and the perceived arousal and mood. It has been used a repeated measures design $(N=27)$. Results show that positive mood increase with high required activation game, meanwhile videogame use doesn't affect negative mood. In regards to perceived arousal, it has been found both low and high required activation videogames there is an increase in perceived arousal. Likewise, there are sex differences in the low required activation videogame. Implications and findings are discussed.

Keywords

videogames; mood; perceived arousal; media psychology; psychological effects 


\section{Introducción}

En los últimos 10 años, la investigación sobre los usos y efectos de los videojuegos ha aumentado considerablemente (Vorderer \& Bryant, 2006). Recurriendo a diferentes metodologías se han estudiado los correlatos cognoscitivos, emocionales, motivacionales y comunicativos, así como las consecuencias sociales del uso de videojuegos. Estamos ante un objeto de estudio altamente cambiante y con una multidimensionalidad en su determinación aún en proceso de definición.

Una de las áreas de investigación importantes en el estudio de los videojuegos es tanto el estudio de su uso sobre el manejo de las emociones como el estudio del lugar de las emociones en el uso de los videojuegos (Bartsch, Mangold, Viehoff, \& Vorderer, 2006; Vorderer, Bryant, Pieper, \& Weber, 2006). Dichos estudios tienen su anclaje en las teorías desarrolladas para la investigación con otros medios (Bryant, Roskos-Edwolsen, \& Cantor, 2003). La investigación acerca de los efectos de los medios sobre los estados de ánimo ha sido una línea permanente en los últimos 25 años, y de sus resultados es de donde ha partido el estudio de los videojuegos. No obstante, como señalan Vorderer y Bryant (2006), su estudio en videojuegos es todavía incipiente.

La investigación sobre los aspectos psicológicos asociados al uso de los videojuegos que permiten explicar el uso mediático, apenas inicia en el contexto costarricense. Estudios como el de Pérez, Rumoroso y Brenes (2009) ofrecen información sobre el aporte de los videojuegos, en el autoconcepto de adolescentes. De igual manera, Brenes (2011) describe el uso generalizado que tienen los videojuegos como activador emocional, específicamente, como medio para la búsqueda de un estado de ánimo positivo, recurriendo a la activación fisiológica que produce este tipo de juego; así se busca olvidar los problemas y evitar la soledad.

Estos efectos se ven diferenciados por sexo. Se han encontrado claras diferencias en el uso de los videojuegos por parte de hombres y mujeres. Los primeros los utilizan con mayor frecuencia y los usan con propósitos competitivos más que las segundas
(Hartmann \& Klimmt, 2007; Lucas \& Sherry, 2004; Williams, 2007). Según Brenes (2011), las mujeres buscan canalizar la agresión física y verbal, mientras que los hombres los utilizan como una estrategia para regular sus emociones negativas.

En este contexto, se considera fundamental indagar en el efecto de los videojuegos sobre el estado de ánimo, recurriendo para ello a lo sistematizado por la teoría del manejo de los estados de ánimo de Dolf Zillmann (1988). Según esta teoría, las personas se orientan a la búsqueda de estados emocionales positivos y a alejarse de lo que le provoque afectos negativos. Los videojuegos les permiten a sus usuarios reacomodar simbólicamente su situación vital; de allí que sean utilizados precisamente como alternativa para modificar sus estados de ánimo, siempre en la búsqueda de afectos más positivos (Oliver, 2003).

Según Zillmann (1988), uno de los predictores de la selección de los contenidos mediáticos es precisamente la regulación de la activación fisiológica. De forma particular, se señala que dado que tanto la sobreestimulación (p. ej., estrés) como la subestimulación (p. ej., aburrimiento) no son estados óptimos, las personas tenderán a escoger contenidos de los videojuegos que le permitan alcanzar estados óptimos (Oliver, 2003). Así, las personas que presenten estados de ánimo atribuidos por ellos mismos como no placenteros tenderán a buscar contenidos que disminuyen estos estados, mientras que quienes presentan estados de ánimos positivos se dirigirán a contenidos que mantengan o aumenten dichos estados.

Durkin y Barber (2002) encuentran que los videojuegos pueden asociarse con la presencia de afectos positivos y con bienestar psicológico. Esto se aplica con más claridad para las personas cuya frecuencia de uso es baja. Este estudio encuentra diferencias por género, pero únicamente en la frecuencia de uso, no en las variables psicológicas asociadas a dicho uso. En un estudio sobre la motivación asociada al uso de videojuegos (Ryan, Rigby, \& Przybylski, 2006), se encontró que aquellas personas que valoran la autonomía y el ser competentes en el uso de videojuegos presentan estados de ánimos positivos después del juego. Esto no parece suceder 
cuando la motivación de logro es la determinante, ya que aparecen más bien afectos negativos. Es posible que estos últimos resultados estén asociados a las características del juego.

Por otro lado, desde la perspectiva de Vorderer, Klimmt y Ritterfeld (2004), las personas usuarias de videojuegos también se dirigen activamente a buscar contenidos que les provoquen afectos negativos (tristeza, miedo, melancolía). Según los autores, esto es posible entenderlo debido a que en la base del uso de estas tecnologías se encuentra el disfrute, y tanto los afectos positivos como los negativos se les asocian. Un elemento que puede explicar este fenómeno son las llamadas metaemociones o metaestados de ánimo, de forma tal que el disfrute puede entenderse como el resultado de la reflexión sobre las emociones que fueron generadas como producto de la interacción con el contenido mediático.

Ravaja, Saari y Salminen (2004) encuentran que, dependiendo de las características del videojuego, se pueden evocar diferentes estados emocionales que pueden estar asociados a la satisfacción de la necesidad de búsqueda de sensaciones, planteada originalmente por Zuckerman (1979), como una de las motivaciones del comportamiento humano. Por ejemplo, Fleming y Rickwood (2001) indagaron en el efecto de un juego de video violento en comparación con uno no violento y otro juego de papel y lápiz, sobre la activación fisiológica y el estado de ánimo positivo y el agresivo; al respecto, se encontró una mayor activación fisiológica ante videojuegos violentos que al resto de juegos. No se encontraron, sin embargo, asociaciones entre el tipo de juego y el estado de ánimo positivo, ni en el estado de ánimo agresivo. En esta misma dirección Barlett y Rodeheffer (2009) encontraron mayor activación fisiológica y sentimientos agresivos ante videojuegos realistas que ante los no realistas o violentos.

Ahora bien, los estudios retoman el efecto de covariación de los estados de ánimo sobre variables como agresión, variaciones en la activación fisiológica o el desempeño en tareas, resultando relativamente escasa la investigación que la evalúa como variable dependiente del juego. Al respecto, Reinecke y Trepte (2008) encontraron que al uso de juegos de computadora se le asocia un aumento en la activación fisiológica, pero además un mejoramiento en la ejecución de tareas de desempeño cognitivo.

En los antecedentes revisados, no se han encontrado estudios que analicen diferentes demandas de activación de los videojuegos sobre la activación fisiológica y el estado de ánimo. Precisamente, el presente estudio se dirige a indagar en la relación entre el uso de videojuegos con diferentes intensidades de activación demandada sobre la activación fisiológica percibida y los estados de ánimo. Se entenderá aquí por intensidad demanda de los videojuegos, 'la intensidad de las demandas de activación motora, de fuerza requerida, de cálculo y coordinación visomotora que requiera el videojuego para ser jugado'.

Las hipótesis del estudio serían las siguientes:

$\mathrm{H} 1$ : El uso de videojuegos provoca un cambio del estado de ánimo hacia afectos más positivos. Este cambio se encuentra relacionado con la intensidad demandada por el videojuego.

$\mathrm{H} 2$ : El uso de videojuegos provoca una disminución en los afectos negativos proporcional a la intensidad de activación demandada por el videojuego.

H3: El efecto sobre los estados de ánimo al usar videojuegos varía según el sexo de la persona usuaria.

H4: El uso de videojuegos provoca un aumento en la activación fisiológica percibida proporcional a la intensidad de activación demandada por el videojuego.

H5: El efecto sobre la activación fisiológica va a variar según el sexo de la persona usuaria.

\section{Método}

\section{Diseño}

Se utilizó un diseño de medidas repetidas 2X3X2 con sexo (hombre y mujer), tiempo de medición (pre y pos) y activación demandada del videojuego (baja, intermedia y alta) como variables independientes, donde la intensidad de activación demandada del videojuego y el tiempo de medición funcionan como variables intrasujeto. 


\section{Participantes}

Los datos fueron tomados de 27 estudiantes universitarios reclutados de cursos de la Escuela de Psicología de la Universidad de Costa Rica; la participación de cada estudiante fue voluntaria. Esta muestra, con un tamaño del efecto medio (0.25), representa un poder estadístico de 0.94 .

Las edades de los participantes oscilaban entre los 18 y 24 años $(M=20.59, D E=1.37) ; 17$ participantes eran mujeres y el $85 \%$ de los participantes reportaron escaso o regular dominio de la consola Wii.

\section{Materiales}

Se decidió escoger los juegos dentro de un mismo formato y género, para evitar que estos elementos afectaran las medidas realizadas. Los juegos fueron escogidos a través del criterio de jueces de diferentes tipos (jugadores de videojuegos, expertos y fisiólogos). Cada uno de estos grupos de jueces evalúo los diferentes minijuegos de Wii Sports Resort en términos de intensidad motora, cálculo, fuerza requerida y coordinación visomotora.

Para este estudio, se utilizaron tres minijuegos del juego Wii Sports Resort de la consola Wii, a saber: frisbee (baja intensidad demandada), golf (intensidad intermedia demandada) y espadas aventura (alta intensidad demandada). Cada uno de estos juegos se trata de una simulación del deporte que se practica; así, frisbee consiste en lanzar un frisbee hacia un blanco, entre más cerca del centro sea lanzado mayor puntaje se obtiene; golf consiste en lanzar la bola en el hoyo en cierto número de tiros y espadas aventura, por su parte, en vencer a los enemigos que se van presentando, para lograr pasar de nivel.

Por su parte, la consola de Wii utiliza un control más intuitivo en el que se simula el movimiento real de los brazos y el cuerpo, ya sea al lanzar el frisbee, utilizar un palo de golf o golpear con una espada. Precisamente, para el juego Wii Sports Resort se introduce el Wii Motion Plus, que es un accesorio que aumenta la precisión del movimiento a la hora de jugar.
Para medir cambios en el estado de ánimo se utilizó la Lista de Afectos Positivos y Negativos (PANAS) desarrollada por Watson, Clark y Tellegen (1988), en la versión al español y adaptación para Costa Rica de Gutiérrez-Doña (2003). Este instrumento consta de dos subescalas con 10 ítems cada una: afecto positivo (con ítems como interesado, orgulloso de mí) y afecto negativo (con ítems como irritable, avergonzado), las cuales constan de una lista de adjetivos que deben calificarse en una escala de 5 puntos según la presencia de cada estado de ánimo (de mucho a nada).

Para este estudio, se obtuvieron coeficientes alfa de Cronbach para la subescala de afecto positivo de 0.9 (condición pre juego baja intensidad), 0.94 (condición pos juego baja intensidad), 0.86 (condición pre juego intensidad media), 0.92 (condición pos juego intensidad media), 0.94 (condición pre juego alta intensidad) y 0.87 (condición pos juego alta intensidad); así como 0.7 (condición pre juego baja intensidad), 0.84 (condición pos juego baja intensidad), 83 (condición pre juego intensidad media), 0.9 (condición post juego intensidad media), 0.74 (condición pre juego alta intensidad) y 0.72 (condición post juego alta intensidad) para la subescala de afecto negativo. Para este estudio, la instrucción original fue modificada, ya que el interés era evaluar el estado de ánimo actual. Esta escala fue diligenciada antes y después de cada sesión de juego.

Por otro lado, para medir los cambios en la activación percibida, se utilizó la Perceived Arousal Scale desarrollada por Anderson, Deuser y DeNeve (1995). Este instrumento consta de 24 ítems que conforman una lista de adjetivos que deben calificarse en una escala de 5 puntos según la presencia de cada estado de ánimo (de mucho a nada), algunos ejemplos de ítems son: "animado" y "lento". Para este estudio, se obtuvieron coeficientes alfa de cronbach de 0.95 (condición pre juego baja intensidad), 0.95 (condición pos juego baja intensidad), 0.89 (condición pre juego intensidad media), 0.92 (condición pos juego intensidad media), 0.97 (condición pre juego alta intensidad) y 0.91 (condición pos juego alta intensidad). Esta escala fue diligenciada antes y después de cada sesión de juego. 


\section{TABLA 1}

Resultados del análisis de varianza para las variables de tipo de juego, tiempo de medición y sexo en la subescala de afectos positivos del PANAS

\begin{tabular}{lcccc}
\hline Fuente & Gl & SS & MS & $F$ \\
\hline Tiempo & 1 & 1.488 & 1.488 & $7.894^{* *}$ \\
Tiempo*Sexo & 1 & 0.222 & 0.222 & 1.177 \\
Error (Tiempo) & 25 & 4.713 & 0.189 & \\
\hline Juego & 2 & 0.34 & 0.17 & 0.411 \\
Juego*Sexo & 2 & 2.488 & 1.244 & 3.009 \\
Error (Juego) & 50 & 20.676 & 0.414 & \\
\hline Tiempo*Juego & 2 & 1.681 & 0.84 & $5.917^{* *}$ \\
Tiempo*Juego*Sexo & 2 & 0.087 & 0.044 & 0.306 \\
Error (Tiempo*Juego) & 50 & 7.102 & 0.142 & \\
\hline
\end{tabular}

Nota. $\mathrm{GL}=$ Grados de libertad, $\mathrm{SS}=$ Square sum, $\mathrm{MS}=$ Mean square; ${ }^{* *} p<0.01$.

Fuente: elaboración propia

\section{Procedimiento}

Los individuos fueron invitados al laboratorio donde se ejecutaba el experimento para tres sesiones de 30 minutos cada una. En la primera sesión, luego de una pequeña introducción al estudio, se les solicitó su consentimiento informado. Luego, para cada una de las tres sesiones se inició con el llenado de un cuestionario. Cada participante jugó 20 minutos cada uno de los juegos, al terminar completaron nuevamente un cuestionario con las mismas medidas del primero. Todas las aplicaciones fueron realizadas en horario de la mañana. La distancia entre cada participación fue de una semana; además, el orden de presentación de los videojuegos fue aleatorizada.

\section{Resultados}

Los análisis que se presentan a continuación se realizaron con pruebas paramétricas en vez de no paramétricas, a pesar de la diferencia en los tamaños de la muestra, debido a que los análisis de las pruebas de homogeneidad de Levene muestran igualdad de varianzas entre los dos grupos en todos los casos; además, el ANOVA de medidas repetidas realiza una corrección ante los tamaños de muestra diferentes, cosa que no realizan los análisis no paramétricos.

\section{Afectos positivos}

Se utilizó el análisis de mediciones repetidas, para comparar el cambio en los estados afectivos al jugar tres tipos diferentes de videojuegos (baja intensidad demandada, intensidad media y alta intensidad) y las diferencias por sexo del participante. Tanto para la subescala de afectos positivos como para la de afectos negativos se realizaron los mismos análisis.

En el caso de los afectos positivos, los resultados evidenciaron un efecto del tiempo en el cambio de los afectos positivos $\left(F_{(1,25)}=7.894, p=0.009, \eta^{2}=\right.$ $0.24)$, no sucede así con el tipo de juego $(p=0.665)$. Tanto el tiempo de medición $(p=0.288)$ como el tipo de juego $(p=0.058$, ) no muestran una mediación del sexo. Sin embargo, el efecto del tiempo en los cambios del afecto positivo se ve mediado por el tipo de juego utilizado $\left(F_{(2,50)}=5.917, p=0.005\right.$, $\left.\eta^{2}=0.191\right)$ (Tabla 1).

Las comparaciones post hoc utilizando la corrección Bonferroni indicaron que los afectos positivos en la condición pos- fueron significativamente mayores $(\mathrm{M}=3.62,95 \% \mathrm{CI}[3.33,3.91])$ que en la condición pre $(\mathrm{M}=3.42,95 \% \mathrm{CI}[3.16,3.68])$, $p=0.009$ (Figura 1), sin encontrarse diferencias para el sexo $(p=0.139)$ ni para el tipo de juego $(p$ $=0.619)$ (Tabla 2). 
TABla 2.

Puntuaciones medias en el estado de afectos positivos en los tres tipos de juego y tiempos de medición

\begin{tabular}{|c|c|c|c|}
\hline Fuente & Medida & M & $D E$ \\
\hline \multirow[t]{2}{*}{ Tiempo } & Pre & $3.422 \mathrm{a}$ & 0.127 \\
\hline & Pos & $3.62 \mathrm{a}$ & 0.139 \\
\hline \multirow[t]{2}{*}{ Sexo } & Hombres & 3.718 & 0.204 \\
\hline & Mujeres & 3.325 & 0.157 \\
\hline \multirow[t]{3}{*}{ Juego } & Baja Intensidad & 3.464 & 0.142 \\
\hline & Intensidad Media & 3.58 & 0.154 \\
\hline & Alta Intensidad & 3.52 & 0.149 \\
\hline \multirow[t]{7}{*}{ Sexo*Juego } & Masculino & & \\
\hline & Baja intensidad & 3.813 & 0.225 \\
\hline & Intensidad Media & 3.615 & 0.245 \\
\hline & $\begin{array}{l}\text { Alta Intensidad } \\
\text { Femenino }\end{array}$ & 3.725 & 0.236 \\
\hline & Baja intensidad & 3.115 & 0.173 \\
\hline & Intensidad Media & 3.545 & 0.188 \\
\hline & Alta Intensidad & 3.315 & 0.181 \\
\hline \multirow[t]{6}{*}{ Sexo*Tiempo } & Masculino & & \\
\hline & Pre & 3.657 & 0.202 \\
\hline & Pos & 3.779 & 0.221 \\
\hline & Femenino & & \\
\hline & Pre & 3.187 & 0.155 \\
\hline & Pos & 3.462 & 0.169 \\
\hline \multirow[t]{9}{*}{ Juego*Tiempo } & Baja Intensidad & & \\
\hline & Pre & 3.398 & 0.137 \\
\hline & Pos & 3.530 & 0.167 \\
\hline & Intensidad Media & & \\
\hline & Pre & 3.59 & 0.143 \\
\hline & Post & 3.57 & 0.183 \\
\hline & Alta Intensidad & & \\
\hline & Pre & 3.278 & 0.177 \\
\hline & Pos & 3.762 & 0.138 \\
\hline \multirow[t]{20}{*}{ Sexo*Juego*Tiempo } & Masculino & & \\
\hline & Baja intensidad & & \\
\hline & Pre & 3.79 & 0.218 \\
\hline & Pos & 3.836 & 0.264 \\
\hline & Intensidad Media & & \\
\hline & Pre & 3.69 & 3.54 \\
\hline & Pos & 3.54 & 0.291 \\
\hline & Alta Intensidad & & \\
\hline & Pre & 3.49 & 0.281 \\
\hline & Pos & 3.96 & 0.218 \\
\hline & Femenino & & \\
\hline & Baja intensidad & & \\
\hline & Pre & 3.006 & 0.167 \\
\hline & Pos & 3.224 & 0.203 \\
\hline & Intensidad Media & & \\
\hline & Pre & 3.49 & 0.174 \\
\hline & Pos & 3.6 & 0.223 \\
\hline & Alta Intensidad & & \\
\hline & Pre & 3.066 & 0.216 \\
\hline & Pos & 3.563 & 0.168 \\
\hline
\end{tabular}

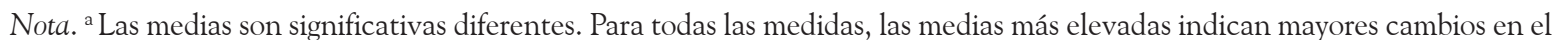
estado afectivo.

Fuente: elaboración propia 


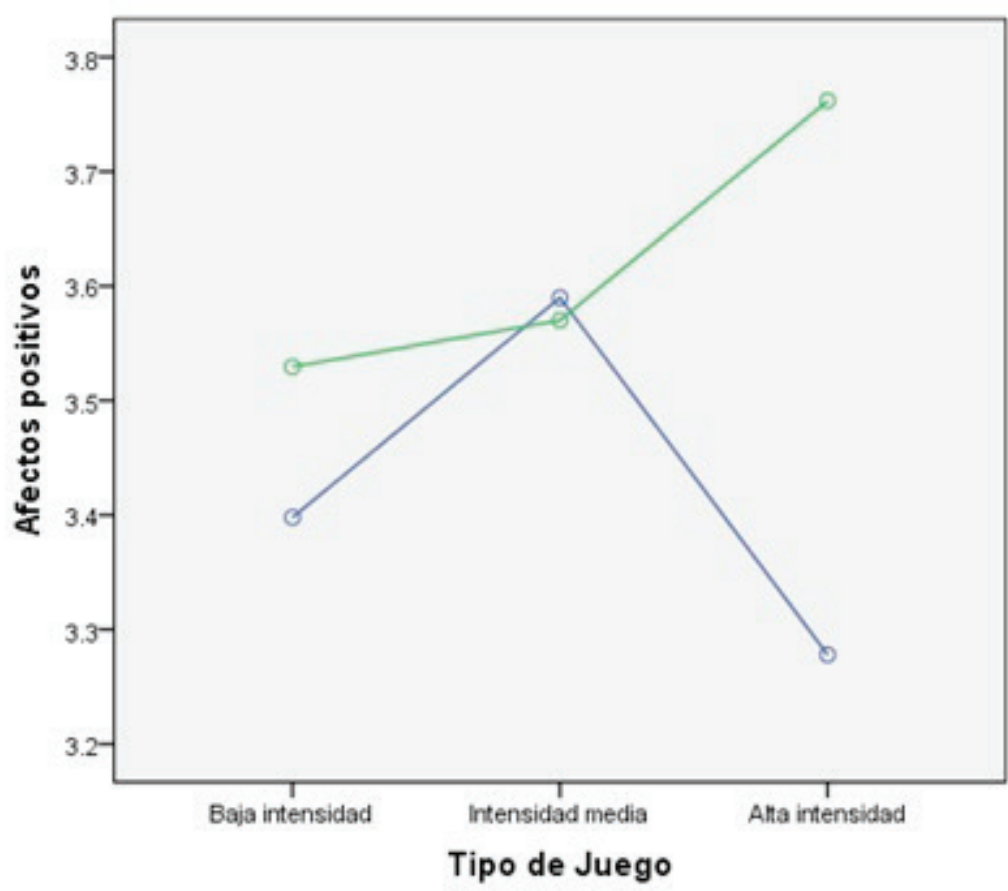

Tiempo de medición

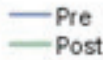

Figura 1. Interacción tiempo de medición por tipo de juego para la escala de afectos positivos del PANAS.

Fuente: elaboración propia

Para analizar la mediación del tipo de juego en los tiempos de medición, se realizó una prueba $t$ para muestras relacionadas, comparando los tiempos de medición para cada uno de los tipos de juegos (Tabla 3). En este análisis, se encontró que el juego de alta intensidad es el que produce una diferencia significativa en los tiempos de medición $\left(t_{26}=-4.661, p<0.001\right)$, con un mayor

\section{TABLA 3}

Resultados del análisis de varianza para las variables de tipo de juego, tiempo de medición y sexo en la subescala de afectos negativos del PANAS

\begin{tabular}{lcccc}
\hline \multicolumn{1}{c}{ Fuente } & Gl & SS & MS & $F$ \\
\hline Tiempo & 1 & 0.115 & 0.115 & 0.854 \\
Tiempo*Sexo & 1 & 0.024 & 0.024 & 0.178 \\
Error (Tiempo) & 25 & 3.368 & 0.135 & \\
\hline Juego & 2 & 0.094 & 0.047 & 0.34 \\
Juego*Sexo & 2 & 0.279 & 0.14 & 1.007 \\
Error (Juego) & 50 & 6.936 & 0.139 & \\
\hline Tiempo*Juego & 2 & 0.044 & 0.022 & 0.414 \\
Tiempo*Juego*Sexo & 2 & 0.249 & 0.124 & 2.316 \\
Error (Tiempo*Juego) & 50 & 2.688 & 0.054 & \\
\hline
\end{tabular}

Nota. $\mathrm{GL}=$ Grados de libertad, $\mathrm{SS}=$ Square sum, $\mathrm{MS}=$ Mean square.

Fuente: elaboración propia 


\section{TABLA 4}

Resultados del análisis de varianza para las variables de juego, tiempo de medición y sexo en la escala de activación percibida

\begin{tabular}{lcccc}
\hline Fuente & Gl & SS & MS & $F$ \\
\hline Tiempo & 1 & 2.699 & 2.699 & $17.606^{* * *}$ \\
Tiempo*Sexo & 1 & 0.49 & 0.49 & 3.233 \\
Error (Tiempo) & 25 & 3.79 & 0.152 & \\
\hline Juego & 2 & 1.643 & 0.822 & 1.686 \\
Juego*Sexo & 2 & 3.161 & 1.617 & $3.243^{*}$ \\
Error (Juego) & 50 & 24.368 & 0.975 & \\
\hline Tiempo*Juego & 2 & 1.026 & 0.513 & $7.981^{* * *}$ \\
Tiempo*Juego*Sexo & 2 & 0.263 & 0.156 & 2.044 \\
Error (Tiempo*Juego) & 50 & 3.213 & 0.064 & \\
\hline
\end{tabular}

Nota. $\mathrm{GL}=$ Grados de libertad, $\mathrm{SS}=$ Square sum, MS $=$ Mean square; ${ }^{*} p<0.05 ;{ }^{* * *} p<0.001$.

Fuente: elaboración propia

puntaje después de jugar $(M=3.71, D E=0.7)$

en comparación a antes de jugar $(\mathrm{M}=3.22, \mathrm{DE}$ $=0.9)$. No se encontraron diferencias en los juegos de baja intensidad $(p=0.165)$ e intensidad intermedia $(p=0.9)$.

\section{Afectos negativos}

En el caso de los afectos negativos, los resultados no evidenciaron efectos significativos del tiempo $(p=$ $0.364)$ ni del tipo de juego $(p=0.765)$. Tampoco se

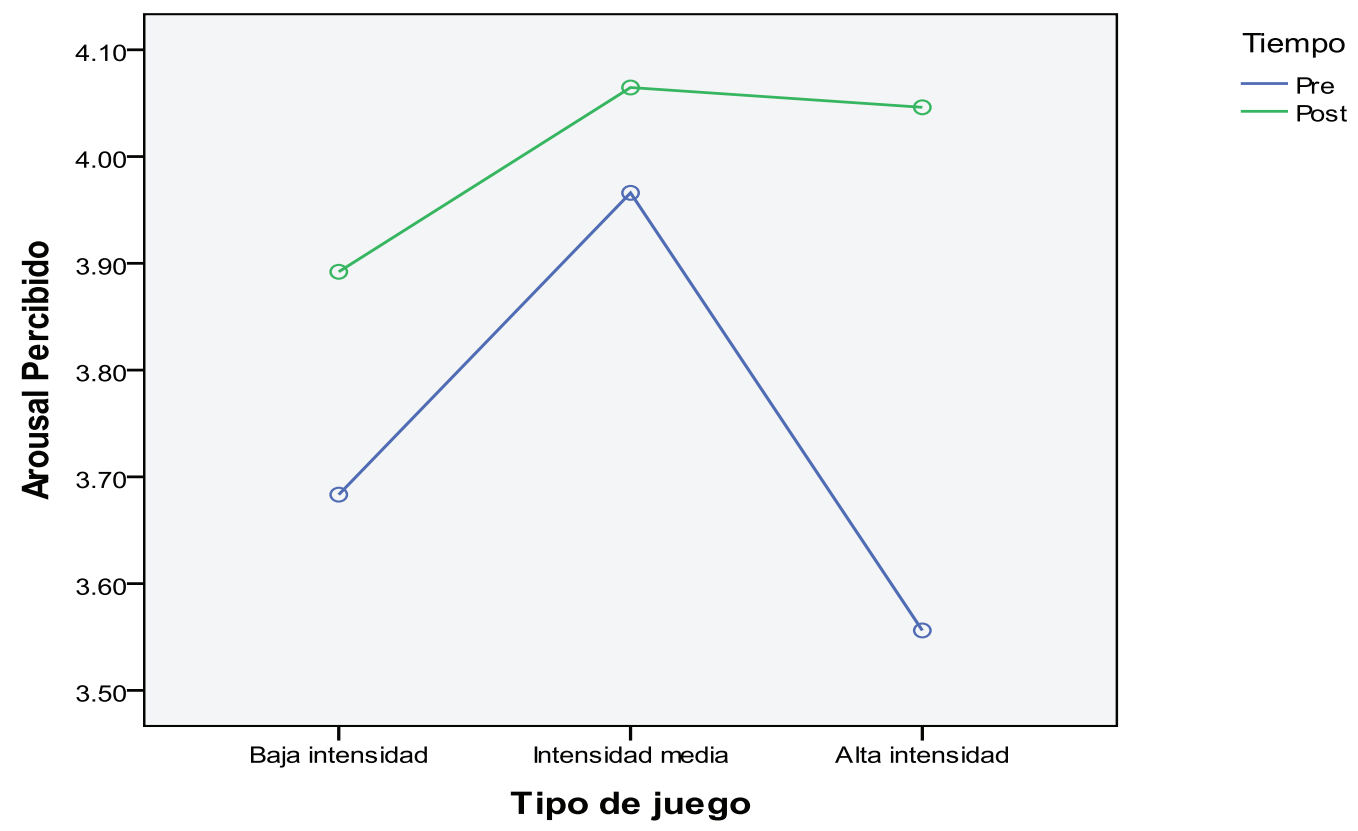

Figura 2. Interacción tiempo de medición por tipo de juego para la activación percibida.

Fuente: elaboración propia 
TABLA 5

Puntuaciones medias en el estado de activación percibida en los tres tipos de juego y tiempos de medición

\begin{tabular}{|c|c|c|c|}
\hline Fuente & Medida & M & $D E$ \\
\hline \multirow[t]{2}{*}{ Tiempo } & Pre & $3.74 a$ & 0.1 \\
\hline & Pos & $4 a$ & 0.09 \\
\hline \multirow[t]{2}{*}{ Sexo } & Hombres & 3.95 & 0.14 \\
\hline & Mujeres & 3.79 & 0.11 \\
\hline \multirow[t]{3}{*}{ Juego } & Baja Intensidad & 3.79 & 0.13 \\
\hline & Intensidad Media & 4.02 & 0.1 \\
\hline & Alta Intensidad & 3.8 & 0.13 \\
\hline \multirow[t]{7}{*}{ Sexo*Juego } & Masculino & & \\
\hline & Baja intensidad & 4.06 & 0.2 \\
\hline & Intensidad Media & 3.95 & 0.16 \\
\hline & $\begin{array}{l}\text { Alta Intensidad } \\
\text { Femenino }\end{array}$ & 3.82 & 0.21 \\
\hline & Baja intensidad & 3.51 & 0.15 \\
\hline & Intensidad Media & 4.08 & 0.12 \\
\hline & Alta Intensidad & 3.78 & 0.16 \\
\hline \multirow[t]{6}{*}{ Sexo*Tiempo } & Masculino & & \\
\hline & Pre & 3.87 & 0.16 \\
\hline & Pos & 4.02 & 0.14 \\
\hline & Femenino & & \\
\hline & Pre & 3.6 & 0.12 \\
\hline & Pos & 3.98 & 0.11 \\
\hline \multirow[t]{9}{*}{ Juego*Tiempo } & Baja Intensidad & & \\
\hline & Pre & 3.68 & 0.12 \\
\hline & Post & 3.89 & 0.13 \\
\hline & Intensidad Media & & \\
\hline & Pre & 3.97 & 0.1 \\
\hline & Post & 4.06 & 0.11 \\
\hline & Alta Intensidad & & \\
\hline & Pre & 3.56 & 0.17 \\
\hline & Post & 4.05 & 0.11 \\
\hline \multirow[t]{20}{*}{ Sexo*Juego*Tiempo } & Masculino & & \\
\hline & Baja intensidad & & \\
\hline & Pre & 4.075 & 0.202 \\
\hline & Post & 4.054 & 0.216 \\
\hline & Intensidad Media & & \\
\hline & Pre & 3.921 & 0.154 \\
\hline & Post & 3.983 & 0.179 \\
\hline & Alta Intensidad & & \\
\hline & Pre & 3.615 & 0.271 \\
\hline & Post & 4.029 & 0.178 \\
\hline & Femenino & & \\
\hline & Baja intensidad & & \\
\hline & Pre & 3.292 & 0.155 \\
\hline & Post & 3.730 & 0.166 \\
\hline & Intensidad Media & & \\
\hline & Pre & 4.011 & 0.118 \\
\hline & Post & 4.146 & 0.138 \\
\hline & Alta Intensidad & & \\
\hline & Pre & 3.497 & 0.208 \\
\hline & Post & 4.063 & 0.136 \\
\hline
\end{tabular}

Nota. ${ }^{\text {LLas }}$ medias son significativas diferentes. Para todas las medidas, las medias más elevadas indican mayores cambios en la activación percibida.

Fuente: elaboración propia 
encontraron mediaciones de sexo en el tiempo de medición $(p=0.677)$ o el tipo de juego $(p=0.361)$, así como tampoco hay efectos en la interacción tiempo de medición*tipo de juego $(p=0.699)$ (Tabla 3).

\section{Activación percibida}

Los resultados evidenciaron un efecto del tiempo en la activación percibida $\left(F_{(1,25)}=17.606, p<0.001, \eta^{2}\right.$ $=0.413)$, no así del tipo de juego utilizado $(p=0.196)$. También se encontró un efecto de la interacción tipo de juego*sexo en la activación percibida $\left(F_{(2,50)}=3.24\right.$, $\left.p=0.047, \eta^{2}=0.115\right)$, no así en la interacción tiempo de medición*sexo $(p=0.084)$. Por otro lado, el efecto del tiempo en los cambios de la activación percibida se ve mediado por el tipo de juego utilizado $\left(F_{(2,50)}=\right.$ $7.981 p<0.001, \eta^{2}=0.242$ ) (Tabla 4).

Las comparaciones post hoc utilizando la corrección Bonferroni indicaron que la activación percibida en la condición pos- fue significativamente mayor $(\mathrm{M}=4,95 \% \mathrm{CI}[3.81,4.19])$ que en la condición pre
$(\mathrm{M}=3.74,95 \% \mathrm{CI}[3.53,3.94]), p=0.002$ (Figura $2)$, sin encontrarse diferencias para sexo $(p=0.395)$ o tipo de juego ( $p=0.361,0.469)$ (Tabla 6 ).

Para analizar la mediación del tipo de juego en los tiempos de medición de la activación percibida, se realizó una prueba t para muestras relacionadas, comparando los tiempos de medición para cada uno de los tipos de juegos (Tabla 7). En este análisis se encontró que tanto el juego de baja intensidad $\left(t_{26}\right.$ $=-3.055, p=0.005)$ como el de alta intensidad $\left(t_{26}=-4.839, p<0.001\right)$ producen una diferencia significativa en los tiempos de medición. No se encontraron diferencias en el juegos de intensidad intermedia $(p=0.071)$.

Por otro lado, para analizar la mediación de sexo en el tipo de juego (Figura 3), se realizó un análisis de varianza simple de las diferencias de la activación percibida para cada uno de los juegos utilizados (Tabla 8). Este análisis muestra que es el juego de baja intensidad $\left(F_{1}=8.125, p=0.009\right)$ el que marca la diferencia significativa del sexo en

\section{TABLA 7}

Diferencias en la activación percibida entre dos tiempos de medición para tres tipos de juego

\begin{tabular}{lccccc}
\hline \multirow{2}{*}{ Tipo de juego } & \multicolumn{3}{c}{ Pre } & \multicolumn{3}{c}{ Pos } \\
\cline { 2 - 7 } & $\mathrm{M}$ & $\mathrm{DE}$ & $\mathrm{M}$ & $\mathrm{DE}$ & $t(26)$ \\
\hline Baja Intensidad & 3.58 & 0.74 & 3.85 & 0.69 & $-3.055^{* *}$ \\
Intensidad media & 3.98 & 0.48 & 4.08 & 0.56 & -1.833 \\
Alta intensidad & 3.54 & 0.84 & 4.05 & 0.55 & $-4.839 * * *$ \\
\hline
\end{tabular}

Nota. ${ }^{* * *} p<0.01{ }^{* * * *} p<0.001$.

Fuente: elaboración propia

\section{TABLA 8}

Resultados del análisis de varianza para el cambio de activación percibida en los tres tipos de juego según sexo

\begin{tabular}{lcccc}
\hline \multicolumn{1}{c}{ Fuente } & Gl & SS & MS & F \\
\hline Juego baja intensidad & & & & \\
Entre grupos & 1 & 1.327 & 1.327 & $8.125^{* *}$ \\
Intragrupos & 25 & 4.084 & 0.163 & \\
Juego intensidad media & & & & 0.361 \\
Entre grupos & 1 & 0.033 & 0.033 & \\
Intragrupos & 25 & 2.278 & 0.091 & 0.475 \\
Juego alta intensidad & 1 & & & \\
Entre grupos & 25 & 0.145 & 0.145 & \\
Intragrupos & 7.643 & 0.306 & \\
\hline
\end{tabular}

Nota. GL = Grados de libertad, SS = Square sum, MS = Mean square; ${ }^{* * *} p<0.01$.

Fuente: elaboración propia 


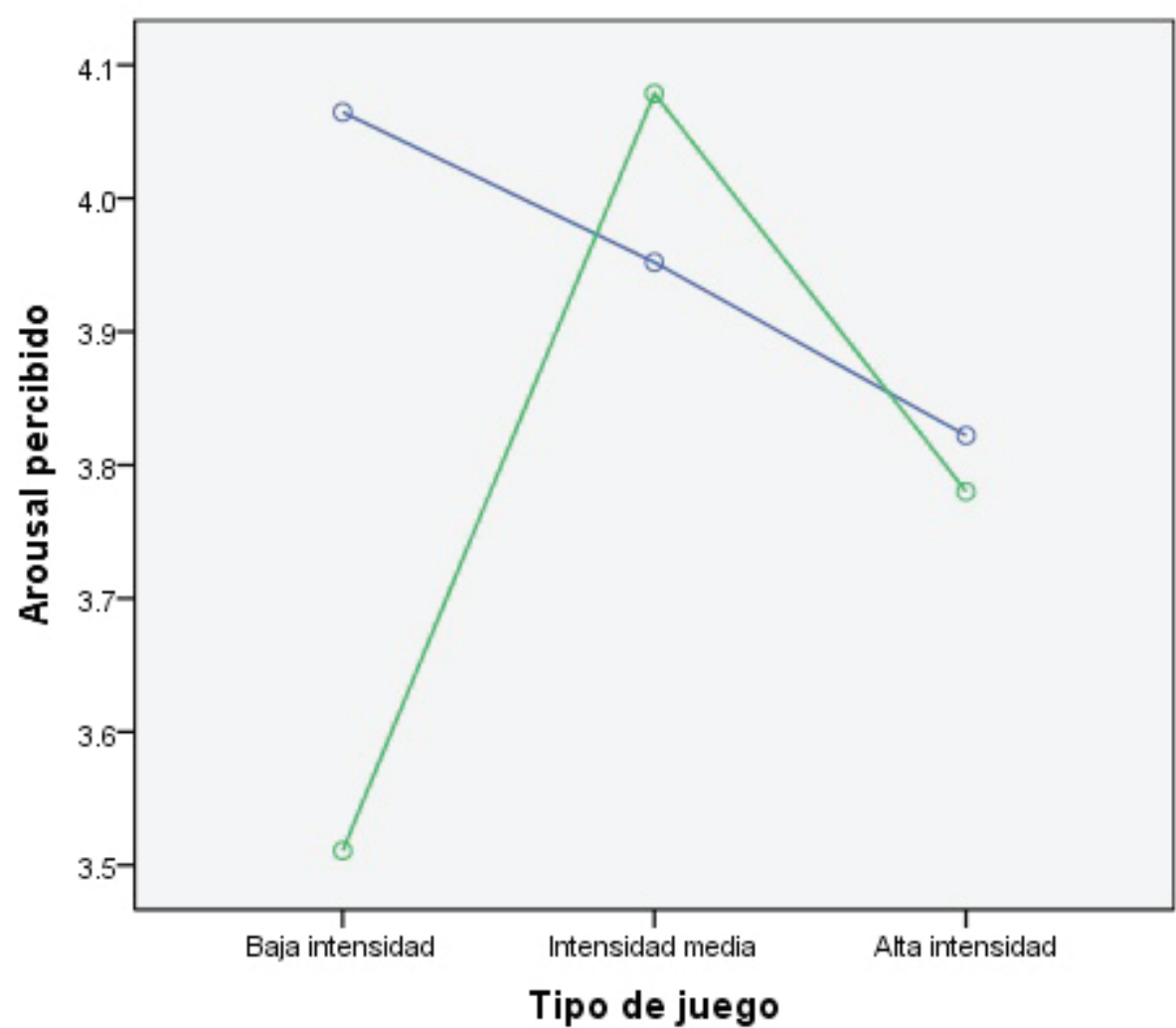

Tipo de juego

Figura 3. Interacción del sexo por tipo de juego para la activación percibida.

Fuente: elaboración propia

el tipo de juego, donde las mujeres muestran un cambio $(\mathrm{M}=0.44,95 \%$ CI $[0.25,0.62])$ en la activación percibida significativamente mayor que los hombres $(M=-0.021,95 \%$ CI $[-0.36,0.31])$; no así el juego de intensidad media $(p=0.553)$ ni el de alta intensidad $(p=0.497)$ (Tabla 9).

\section{Discusión}

El juego de alta intensidad favorece un aumento de los afectos positivos, no así de los afectos negativos, en donde no se evidenció cambio. Únicamente, en esta condición fue donde se encontraron diferencias

\section{TABLA 9}

Medias y desviaciones estándar para sexo y tres tipos de juego

\begin{tabular}{lcccc}
\hline \multirow{2}{*}{ Tipo de juego } & \multicolumn{2}{c}{ Hombre } & $\mathrm{c}$ & Mujer \\
\cline { 2 - 5 } & $\mathrm{M}$ & $\mathrm{DE}$ & 0.44 & $\mathrm{DE}$ \\
\hline Baja Intensidad & -0.21 & 0.47 & 0.13 & 0.36 \\
Intensidad media & 0.06 & 0.31 & 0.57 & 0.3 \\
Alta intensidad & 0.41 & 0.42 & 0.62 \\
\hline
\end{tabular}

Nota. ${ }^{* * *} p<0.01,{ }^{* * *} p<0.001$.

Fuente: elaboración propia 
en el estado de ánimo. No se encontraron diferencias por género.

Estos resultados respaldan la hipótesis 2 de este estudiom, no así la 1 y la 3, lo que implica que el juego por sí solo no va a aumentar los afectos positivos, sino solo los que demandan mayor intensidad. Además de que estos resultados se van a presentar independientemente del género de la persona usuaria. El hecho de que en el caso de los afectos negativos no se haya presentado ninguna variación, puede respaldar el papel del uso de los videojuegos en los afectos positivos

Al respecto, Ravaja et al. (2004) señalan que una de las motivaciones principales del uso de videojuegos es la búsqueda de sensaciones, al igual que lo propone Zuckerman (1979) al hablar de la gratificación de búsqueda de sensaciones, cuando afirma que las personas buscan emociones más allá del nivel óptimo de activación fisiológica percibida. El aumento en los afectos positivos que acontece en el contexto experimental puede estar asociado con estas motivaciones y con la función misma que tiene el juego y el entretenimiento como recurso para la búsqueda de bienestar psicológico, en un momento dado.

Este punto muestra concordancia con lo mencionado por Bartsch et al. (2006) con respecto a que las emociones pueden ser gratificantes, porque ayudan a mantener un estado placentero de activación fisiológica percibida o porque permiten conseguir experiencias novedosas, intensas y sensacionales, además de estar asociadas a habilidades de afrontamiento que conducen a sentimientos de éxito y competencia, si la persona logra afrontar exitosamente una situación.

Es importante considerar, sin embargo, que este aumento en los afectos positivos ante el juego de alta intensidad puede estar asociado a las características del juego mismo. Se trataba de juegos no agresivos, en los que se competía con el propio desempeño y con énfasis en la actividad motora y perceptual. Al respecto, es necesario comparar estos resultados con otros tipos de juego y con situaciones de juego en el que están implicados más de un jugador.

Para el caso de la activación fisiológica percibida, tanto en el juego de baja intensidad como en el juego de alta intensidad demandada se encontró un aumento de dicha activación después del juego. Asimismo, se encontró una mayor activación percibida en el caso de las mujeres respecto a los hombres, después del juego de baja intensidad demandada.

Estos resultados llevan a descartar, al menos para el presente estudio, la hipótesis 4, ya que la activación fisiológica percibida no se presentó como proporcional a la activación demandada por el juego. Por otra parte, la hipótesis 5 se presenta respaldada, sugiriéndose una relación entre la activación fisiológica y el sexo de la persona usuaria.

Un aspecto importante en este punto es que aunque las mujeres presentaron un aumento en su activación fisiológica percibida en el juego de baja intensidad, fueron los hombres lo que percibieron una mayor activación, tanto antes como después del juego.

Sin embargo, otro aspecto importante de notar es que fue en este juego el único donde hubo una disminución en la activación percibida, en este caso para los hombres, aunque la misma no fuera significativa. Una posible explicación a este fenómeno está en la intensidad de uso de los videojuegos en general, con la cual se ha encontrado que los hombres tienden a utilizar más videojuegos que las mujeres (Lucas \& Sherry, 2004). Esta sería una hipótesis necesaria de comprobar en estudios posteriores.

Por otro lado, una hipótesis que puede explicar estas diferencias por sexo puede ser el nivel del significado simbólico de la comunicación emocional, mencionado por Bartsch et al. (2006), en el que las emociones pueden ser gratificantes por su significado en términos de las normas y valores, punto que hace referencia hacia la socialización de las emociones, la cual le da una construcción selectiva al significado emocional relacionado con la experiencia del usuario, como una forma de mostrar sus valores personales, rasgos de carácter deseados y otras características que definen la identidad y estatus social del individuo.

Así, puede que los hombres se encuentren más socializados a elevar su activación durante el uso de videojuegos, a diferencia de las mujeres, por esto, se presentan mayores niveles de activación fisiológica percibida en el uso general del videojuego. 
Por otro lado, las mismas características del juego pueden estar ligadas con los resultados. Así, el juego de baja intensidad de activación demandada requiere, sin embargo, de precisión perceptual, aspecto que pudo provocar activación fisiológica debido a la demanda de los recursos cognoscitivos implicados en esta actividad. En el caso del juego de alta intensidad, la actividad demandada era fundamentalmente motora. En este contexto, resultan interesantes las diferencias encontradas por géneros. Las mujeres respondieron a los requerimientos perceptivos propios del juego de baja intensidad, pese que la demanda de activación motora era mínima respecto al juego de alta intensidad, actividad que no se presentó en los hombres.

Los resultados encontrados tanto para el caso de los estados de ánimo como de la percepción de la activación pueden entenderse en el marco del modelo de la experiencia del entretenimiento expuesto por Vorderer et al. (2004). Así, como ya se ha indicado, los videojuegos a los que se expusieron los participantes fueron percibidos como agentes de activación fisiológica, que fue asociada con afectividad positiva. Acá el disfrute promovido por la situación de juego se vinculó con estados de ánimo placenteros, situaciones que implicaban poner a prueba las destrezas de coordinación visomotora y el competir consigo mismo por la mejoría de estas destrezas (como en el caso del juego de frisbee) o el competir ante personajes virtuales mediante la imposición de fuerza y movimiento en una lucha de espadas.

\section{Referencias}

Anderson, C. A., Deuser, W. E., \& DeNeve, K. (1995). Hot temperatures, hostile affect, hostile cognition, and arousal: Tests of a general model of affective aggression. Personality and Social Psychology Bulletin, 21(5), 434-448.

Barlett, C., \& Rodeheffer, C. (2009). Effects of realism on extended violent and nonviolent video game play on aggressive thoughts, feelings, and physiological arousal. Aggressive Behavior, 35(3), 213-224.

Bartsch, A., Mangold, R., Viehoff, R., \& Vorderer, P. (2006). Emotional gratifications during media use: An integrative approach. Communications; The European Journal of Communication Research, 31(3), 261-278.

Brenes, C. (2011). Dimensiones asociadas al uso de los video-juegos en niños y niñas escolares. En R. Pérez (Ed.), Psicología de los usos de los medios. Avances en la investigación de la psicología de los medios de comunicación (pp. 94-136). San José: IIP.

Bryant, J., Roskos-Ewoldsen, D. \& Cantor, J. (2003). Communication and emotion: Essays in honor of Dolf Zillmann. Mahwah, NJ: Lawrence Erlbaum Associates.

Durkin, K., \& Barber, B. (2002). Not so doomed: Computer game play and positive adolescent development. Journal of Applied Developmental Psychology, 23(4), 373-392.

Fleming, M., \& Rickwood, D. (2001). Effects of violent versus nonviolent video games on children's arousal, aggressive mood, and positive mood. Journal of Applied Social Psychology, 31(10), 2047-2071.

Gutiérrez-Doña, B. (2003). Coping with stress at work: A longitudinal study on health outcomes and quality of life (Fachbereich Erziehungswissenschaft und Psychologie der Freien Universität Berlin. Dissertation zur Erlangung des akademischen Grades Doktor Der Philosophie). Recuperado de http://www.diss. fu-berlin.de/diss/servlets/MCRFileNodeServlet/ FUDISS_derivate_000000000898/

Hartmann, T., \& Klimmt, C. (2006).The influence of personality factor on computer games choice. En P. Vorderer \& J. Bryant (Eds.), Playing video games: Motives, responses, and consequences (pp. 115133). New Jersey: Erlbaum.

Lucas, K., \& Sherry, J. L. (2004). Sex differences in video game play: a communication-based explanation. Communication research, 31(5), 499-523.

Oliver, M. (2003). Mood management and selective exposure. En J. Bryant, D. Roskos-Edwolsen \& J. Cantor (Eds.), Communication and emotion essays in honor of Dolf Zillmann (pp. 83-106). New Jersey: Erlbaum.

Pérez, R., Rumoroso, A., \& Brenes, C. (2009). El uso de tecnologías de la información y la comunicación y la evaluación de sí mismo en adolescentes costarricenses Revista Interamericana de Psicología, 43(3), 610-617. 
Ravaja, M., Saari, T., \& Salminen, M. (2004). Emotional response patterns and sense of presence during video games: Potential criterion variables for game design. Proceedings of the Third Nordic Conference on Human-Computer Interaction (pp. 339-347). Tampere, Finland: Association of Computing Machinery Press.

Reinecke, L., \& Trepte, S. (2008). In a working mood? The effects of mood management processes on subsequent cognitive performance. Journal of Media Psychology, 20(1), 3-14.

Ryan, R. M., Rigby, S., \& Przybylski, A. (2006). The motivational pull of video games: A self-determination theory approach. Motivation and Emotion, 30(4), 344-360.

Vorderer, P., \& Bryant, J. (2006). Playing videogames: Motives, responses, and consequences. London: Routledge.

Vorderer, P., Bryant, J., Pieper, K., \& Weber, R. (2006). Playing video-games as entertainment. En P. Vorderer \& J. Bryant (Eds.), Playing video games:
Motives, responses, and consequences (pp. 1-8). New Jersey: Erlbaum.

Vorderer, P., Klimmt, C., \& Ritterfeld, U. (2004). Enjoyment: At the heart of media entertainment. Communication Theory, 14(4), 388-408.

Watson, D., Clark L.A., \& Tellegen, A. (1988). Development and Validation of Brief Measures of Positive and Negative Affect: The PANAS Scales. Journal of Personality and Social Psychology, 54(6), 1063-1070.

Williams, D. (2007). A brief social history of game play. En P. Vorderer \& J. Bryant (Eds.), Playing video games: Motives, responses, and consequences (pp. 197-212). New Jersey: Erlbaum.

Zillmann, D. (1988). Mood management: Using entertainment to full advantage. En L. Donohew, $\mathrm{H}$. E. Sypher \& E. T. Higgins (Eds.), Communication, social cognition, and affect (pp. 147-171). New Jersey: Erlbaum.

Zuckerman, M. (1979). Sensation seeking: Beyond the optimal level of arousal. New York: Wiley. 